Brit. J. industr. Med., 1956, 13, 214.

\title{
TWO CASES OF POISONING WITH DI-ISOPROPYLFLUOROPHOSPHONATE (D.F.P.)
}

\author{
BY
}

\author{
W. K. S. MOORE \\ From the Industrial Health Unit, Boots Pure Drug Co. Ltd., Nottingham
}

(RECEIVED FOR PUBLICATION OCTOBER 28, 1954)

Di-isopropylfluorophosphonate (D.F.P.) has the property of inhibiting the enzyme cholinesterase, and it has been recommended for use in the treatment of glaucoma, paralytic ileus, and myasthenia gravis. It is manufactured on a laboratory scale as this is sufficient to produce the quantities required for clinical use.

The circumstances in which two chemical workers were intoxicated with D.F.P. are reported together with some clinical details of their illnesses.

\section{Method of Manufacture}

Di-isopropylfluorophosphonate is prepared in three stages, the last of which involves the formation and isolation of D.F.P. by distillation from the reaction mixture. It is only the third stage which carries any risk of poisoning.

During this stage the area of the laboratory and its environs are made "out of bounds". The apparatus is erected in a fume cupboard where all manipulations are carried out. There are always three people present during the third stage, only one of whom requires to approach the fume cupboard, the other two standing by to give assistance in any emergency. All three workers wear rubber gloves, and, in addition, a respirator is put on before handling the apparatus in the fume cupboard. At the end of the process the apparatus is contaminated with traces of D.F.P. and the distillation flask itself contains a high boiling oily residue which may contain an appreciable amount of D.F.P. which has failed to distil over. For this reason, all the equipment used in the third stage must be treated with alkali in order to decompose any residual D.F.P. This is done by placing all the apparatus in a large vessel containing sodium carbonate solution for at least 48 hours before washing with water and drying.

\section{History of Exposure}

Di-isopropylfluorophosphonate was manufactured using these precautions during the week beginning
Monday, November 9, 1953. When the preparation was completed on Friday, November 13, all the apparatus which had been used was placed in a large pan containing aqueous sodium carbonate solution and left during the weekend. The oily residue from the distillation sank to the bottom of the aqueous solution, and amounted to some $250 \mathrm{~g}$.

On Monday, November 16, the sodium carbonate solution was syphoned out of the pan and replaced by water. This wash was repeated with another change of water. The apparatus was then removed to be washed and dried. No hand protection was used in this work. Certain pieces of apparatus were immersed in the oily layer at the bottom of the pan, and the workers' hands had to enter the oil in order to recover these components. Two men were engaged in this task, one of whom (G. H. S.) recovered most of the apparatus from the pan, while the other (T. H.) helped with washing and drying the glassware. They began work at 9.45 a.m. on November 16. At about 11 a.m. on the same morning G. H. S. noticed that the laboratory appeared to have become dimly lit, and for this reason he reported to the Surgery. T. H. did not notice any symptoms until he got home from work in the evening.

\section{Case Histories}

Case 1.-G. H. S. reported to the Surgery on November 16 at 11 a.m. He was 26 years of age, and had been employed by the firm since March, 1948. There was nothing relevant in his previous medical history. At his pre-placement medical examination his visual acuity was found to be $\mathrm{R} \mathrm{V} \mathrm{6/24} \mathrm{J} \mathrm{5,} \mathrm{L} \mathrm{V} \mathrm{6/6} \mathrm{J} \mathrm{1.} \mathrm{No} \mathrm{other} \mathrm{abnor-}$ malities were noted.

He complained of dim vision, difficulty in focusing on near objects, and a tendency for his eyes to water. On examination his pupils were seen to be very small and they did not react to light or accommodation.

He was given a tepid alkaline bath and rested in the Surgery. At 2.30 p.m. he felt quite well, but still had 
miosis. He was sent home by car. During the evening he noticed some pain behind the eyes. Between midday on November 16 and the morning of November 17 he had three bowel motions, which was unusually frequent.

After breakfast on November 17 he experienced nausea, his vision remained dim, and accommodation for near objects was lacking. There were no respiratory symptoms. When he was seen on November 17, the pupils were small and fixed. The finger-nose test was carried out inaccurately while his eyes were open, but improved in performance if he shut his eyes. The tendon jerks were brisk and equal. A blood sample was taken for cholinesterase determination.

He was given an intravenous injection of atropine, gr. $1 / 30$, at midday. At 2.15 p.m. the pupils were dilated, and gave a small response to light. His mouth felt very dry, but by 3.30 p.m. moisture returned in his mouth. About this time he found that his stream of urine slowed and stopped during micturition, so that he had to make an effort to start again. By 6 p.m. this last symptom disappeared, and at the same time his vision suddenly became dim again.

On November 18 he had nausea after breakfast. His vision was dim, but he had infrequent periods of brightening during the day, and during these periods he could focus clearly on near objects. During the afternoon he felt very depressed for about three hours, and he was afraid that his vision would never become normal. At 9.45 p.m. his vision suddenly became brighter.

On November 19 his vision remained bright except for short periods when the dimming returned. On examination the pupils were less contracted, but the right pupil was a little larger than the left. The finger-nose test was quite normal.

On November 23 his vision was almost normal. The pupils were still unequal but reacted to light. He had the impression that his recovery had occurred in sudden steps rather than in a continuous and gradual way. Three months later his pupils remained unequal in size, but his visual acuity was the same as that recorded at his pre-placement examination.

CHOLINESTERASE ESTIMATIONS*

\begin{tabular}{c|c|c}
\hline Date & Plasma (units) & R.B.C. (units) \\
\hline 17.11 .53 & 34 & 101 (Warburg method) \\
7.12 .53 & 97 & 110 (Warburg method) \\
$\mathbf{2 8 . 1 2 . 5 3}$ & 121 & 114 (Warburg method) \\
\hline
\end{tabular}

* Normal values are about 100 units in both plasma and red cells.

Case 2.-T. H., aged 20 years, had been employed by the Company since 1949 . No significant abnormalities were noted at his pre-employment examination, and there was no relevant medical history.

He helped G. H. S. to wash and clean apparatus on November 16, 1953, but so far as he could remember he did not touch the oily sediment in the vessel containing the apparatus. During the evening he noticed that the room he was in had become darker. On the morning of November 17 he had nausea and then he vomited. Con- trary to custom he had three bowel motions between midday on November 16 and the morning of November 17. He experienced no respiratory symptoms, nor did he have pain in the eyes.

When he reported to the Surgery on November 17 the pupils were contracted and gave no reflex reaction to light or accommodation. He was given a tepid alkaline bath. A sample of blood was taken for cholinesterase estimation.

On November 19 his vision was almost normal, but he noticed that it was slow in adapting for brighter levels of illumination. His pupils were no longer contracted, and pupillary reflex reactions were normal.

CHOLINESTERASE ESTIMATIONS

\begin{tabular}{c|c|c}
\hline Date & Plasma (units) & R.B.C. (units) \\
\hline 17.11 .53 & 49 & 115 (Warburg method) \\
7.12 .53 & 81 & 115 (Warburg method) \\
28.12 .53 & 91 & 117 (Warburg method) \\
\hline
\end{tabular}

\section{Discussion}

Although there are obvious risks connected with the manufacture of D.F.P., no case of poisoning had occurred in this firm until the incident described here.

In this instance, the source of D.F.P. was an oily residue from the final distillation in the process, which lay at the bottom of a large pan under an aqueous layer of sodium carbonate solution. Various components of the apparatus which had been immersed in the oily residue were handled while being cleaned by the men who became affected.

It seems certain that the men's hands must have become contaminated with the D.F.P.-containing residue, and a certain amount must have been absorbed through the skin, thus causing a fall in the cholinesterase of the plasma. Di-isopropylfluorophosphonate, however, is a selective inhibitor of pseudo-cholinesterase (Hawkins and Mendel, 1947) which constitutes $99 \%$ of the cholinesterase content of human plasma (Mendel, Mundell, and Rudney, 1943) and it has been found that almost complete inhibition of the cholinesterase activity of human plasma can be produced with D.F.P. without causing serious distress (Bodansky, 1945; Mazur and Bodansky, 1946).

In the cases described here, two-thirds and a half of the plasma cholinesterase activity was lost, but these deficits are unlikely to be associated with systemic effects of acetylcholine poisoning. On the other hand, both men suffered alimentary symptoms as well as pupillary changes, and although the latter might be explained by assuming accidental introduction of D.F.P. to the eyes by hand or vapour, neither of these workers would be likely to put their hands to their mouths so producing direct contamination of the alimentary tract. 
It is impossible to be certain of the exact sequence of events, and further discussion could only be based on assumption.

Once it was realized that the residue from the distillation in the last stage of the manufacturing process might contain dangerous quantities of D.F.P., a method of decontamination was devised, whereby further additions of sodium carbonate solution were stirred into the residue. The decomposition of D.F.P. is accompanied by effervescence; sodium carbonate solution is added so long as this continues.
Full protective clothing, including gloves, respirator, and gown, are worn during this procedure.

The cholinesterase estimations were carried out by Dr. E. N. Allott, Group Laboratory, Lewisham Hospital, London, S.E.11, to whom I am indebted.

\section{REFERENCES}

Bodansky, O. (1945). Science, 102, 517.

, and Mazur, A. (1946). Fed. Proc., 5, 123.

Hawkins, R. D., and Mendel, B. (1947). Brit. J. Pharmacol., 2, 173.

Mendel, B., Mundell, D. B., and Rudney, H. (1943). Biochem. J., 37, 473 . 